\title{
Qualitative detection of single submicron and nanoparticles in human skin by scanning transmission $\mathrm{x}$-ray microscopy
}

Christina Gra删

Freie Universität Berlin

Physikalische und Theoretische Chemie

Takustr. 3, 14195 Berlin, Germany

\author{
Martina Meink *† $^{*}$ \\ Charité-Universitätsmedizin Berlin \\ Department of Dermatology and Allergy \\ Center of Experimental and Applied Cutaneous \\ Physiology \\ Chariteplatz 1, 10117 Berlin, Germany
}

\section{Qi Gao}

Freie Universität Berlin

Physikalische und Theoretische Chemie

Takustr. 3, 14195 Berlin, Germany

\section{Sabrina Hadam}

Charité-Universitätsmedizin Berlin

Clinical Research Center for Hair and Skin Science

Department of Dermatology and Allergy

Chariteplatz 1, 10117 Berlin, Germany

\section{Jörg Raabe}

Paul Scherrer Institut

Swiss Light Source

5232 Villigen PSI, Schweitz, Switzertand

\author{
Wolfram Sterry \\ Charité-Universitätsmedizin Berlin \\ Department of Dermatology and Allergy \\ Center of Experimental and Applied Cutaneous \\ Physiology \\ and \\ Clinical Research Center for Hair and Skin Science \\ Department of Dermatology and Allergy \\ Chariteplatz 1, 10117 Berlin, Germany

\section{Ulrike Blume-Peytavi} \\ Charité-Universitätsmedizin Berlin \\ Clinical Research Center for Hair and Skin Science \\ Department of Dermatology and Allergy \\ Chariteplatz 1, 10117 Berlin, Germany
}

\section{Jürgen Lademann}

Charité-Universitätsmedizin Berlin

Department of Dermatology and Allergy

Center of Experimental and Applied Cutaneous

Physiology

Chariteplatz 1, 10117 Berlin, Germany

\footnotetext{
${ }^{\dagger}$ Both authors contributed equally to this work.

*Address all correspondence to: Christina Graf, Freie Universität Berlin, Physikalische und Theoretische Chemie, Takustr. 3, 14195 Berlin, Germany, E-mail: cmgraf@chemie.fu-berlin.de; or, Annika Vogt Charité-Universitätsmedizin Berlin, Clinical Research Center for Hair and Skin Science, Department of Dermatology and Allergy, Charitéplatz 1, 10117 Berlin, Germany, E-mail: annika.vogt@charite.de.
}

\section{Eckart Rühl}

Freie Universität Berlin

Physikalische und Theoretische Chemie

Takustr. 3, 14195 Berlin, Germany

\author{
Annika Vogt \\ Charité—Universitätsmedizin Berlin \\ Clinical Research Center for Hair and Skin Science \\ Department of Dermatology and Allergy \\ Chariteplatz 1, 10117 Berlin, Germany
}

Abstract. First results on single particle detection in human skin samples by $\mathrm{x}$-ray microscopy are reported. $94 \pm 6$ and $161 \pm 13 \mathrm{~nm}$ gold core particles with silica shells and $298 \pm 11 \mathrm{~nm}$ silica particles coated with a gold shell on ultramicrotome sections of human skin were determined. The particles were applied on fresh intact skin samples, which were sectioned prior to imaging. After screening the sections by conventional microscopy techniques, defined areas of interest were qualitatively investigated by scanning transmission x-ray microscopy at the Swiss Light Source. In studies on the percutaneous penetration of $161 \pm 13 \mathrm{~nm}$ particles on human skin samples, $\mathrm{x}$-ray microscopy yielded high-resolution images of single particles spreading on the superficial layer of the stratum corneum and on the epithelium in superficial parts of hair follicles. No deeper penetration was observed. The present work using x-ray microscopy provides the unique opportunity to study qualitative penetration processes and membrane-particle interactions on the level of single particles. This goes beyond present approaches using optical microscopy. Further improvement of this approach will allow one to study particles with different physicochemical properties and surface modifications, including responses of the exposed tissue. ( $) 2009$ Society of Photo-Optical Instrumentation Engineers. [DOI: 10.1117/1.3078811]

Keywords: percutaneous penetration; hair follicle; nanotechnology; x-ray microscopy; synchrotron radiation; nanoparticles.

Paper 08213SSRR received Jul. 7, 2008; revised manuscript received Oct. 31, 2008; accepted for publication Nov. 15, 2008; published online Mar. 4, 2009

\section{Introduction}

With the increased use of nanoparticles in technical and biomedical applications, such as sunscreens, body lotions, functional textiles, and tumor diagnostics, the investigation of possible hazardous effects related to intended and unintended nanoparticle exposure has become an important field of research The skin, with an average surface of $1.5-2 \mathrm{~m}^{2}$ in human adults, provides a large surface for nanoparticle-cell interactions and hence represents an important putative site for nanoparticle translocation into the viable tissue. General

1083-3668/2009/14(2)/021015/9/\$25.00 @ 2009 SPIE 
percutaneous absorption can be described by intercellular stratum corneum or via follicular penetration 2 Recent investigations have demonstrated the important role of skin appendages, above all hair follicles, as the entry point and reservoir for topically applied compounds 3 This penetration route is especially favored by particle formulations, which is due to the ability to aggregate in the hair follicle openings and to penetrate along the follicular duct. In fact, storage of particles of a size of $320 \mathrm{~nm}$ in the follicular duct was monitored in vivo for up to 10 days, providing enough time for intensive interactions between environmental and product-related nanoparticles with the epithelium or associated cell populations, respectively 4

Physicochemical parameters of the nanoparticles of a given chemical composition, such as size, charge, and lipophilicity, as well as pretreatment techniques (e.g., massage, local heating, and stripping techniques) were shown to influence the percutaneous penetration process 57 Stripping techniques, such as tape stripping and cyanoacrylate skin surface stripping, disturb the skin barrier and facilitate the penetration of topically applied substances 58 However, the vast majority of studies were performed on micro- and nanoparticles sized $>750 \mathrm{~nm}$ that showed no penetration into viable tissue. Only recently, research shifted toward smaller particle sizes. At present, there is little evidence that nanoparticles at a size exceeding $100 \mathrm{~nm}$ penetrate into intact skin! 1 If particles $<100 \mathrm{~nm}$ penetrated into intact skin remains unclear. Cross et al., for example, reported rather minimal penetration of 26-30 nm micronized zinc oxide into the upper layers of the stratum corneum of human skin 9 while Baroli et al. suggest that metallic nanoparticles as small as $5.9 \mathrm{~nm}$ penetrated intact stratum corneum and hair follicles in a diffusion cell model of excised human skin 10 The relevance of nanoparticle translocation across the skin barrier becomes even more apparent by investigations on particle penetration in barrierdisrupted or otherwise damaged skin as well as the possibility of shunt penetration via skin appendages. In our own studies on the penetration of polystyrene particles in human skin pretreated with cyanacrylate skin surface stripping, which induced mild barrier disruption and opens hair follicles for penetration, we found that $40-\mathrm{nm}$, but not 750 or $1500-\mathrm{nm}$ particles penetrated, most likely via hair follicles, and were internalized by epidermal Langerhans cells 11 In accordance with these results, Zhang et al. found penetration of quantum dot nanoparticles into the outer root sheath (e.g., the viable epithelial layers of hair follicles), while interfollicular penetration was limited to the upper layers of the stratum corneum 12 Similarly, flexing of porcine skin significantly increased penetration of 3.5-nm fullerene amino acidderivatized peptide nanoparticles 13

Microscopy techniques utilizing visible light, such as standard optical microscopy, fluorescence microscopy, and confocal laser scanning microscopy, were used in the past to visualize the uptake of nanoparticles into skin 14.16 Direct imaging of single nanoparticles is not possible with such techniques because their size is below the diffraction limit. Scanning transmission X-ray microscopy 17 (STXM) has been used for the investigation of cells in the last few years 2022 Various studies devoted to materials sciences (e.g., on hollow microcapsules and core-shell polymer micro-spheres) have also made use of x-ray microscopy 23 In STXM, high-brilliance synchrotron radiation is tightly focused $(40 \mathrm{~nm})$, and the sample is raster scanned while recording the intensity of transmitted x-rays in order to produce a two-dimensional image. Besides from spatial resolution, x-ray microscopy also provides chemical contrast, which is a result of strong variations of the absorption cross section in core level absorption. This is due to the distinct near-edge x-ray absorption fine structure In this way, the combination of spectroscopic and ultrastructural data provides at $\sim 40 \mathrm{~nm}$ spatial resolution information of the chemical composition of the sample. Therefore, x-ray microscopy has significant advantages compared to fluorescence microscopy techniques besides chemical contrast and sub-40-nm spatial resolution, because even wet samples without staining or using markers can be studied 28 STXM has only been applied to a limitednumber of biological or biomedical samples in the past 212835 To the best of our knowledge, the interaction of particles in the submicron and nanometer range with human skin samples has not been investigated by STXM. Transmission electron microscopy (TEM) has already been used to localize the $\mathrm{TiO}_{2}$ nanoparticles in skin sections, but this technique requires ultrathin skin sections $(50 \mathrm{~nm})$ and staining of the samples 36 Ultrasections prepared after skin-penetration experiments have to be significantly thicker than the particles under investigation to prevent that the particles are lost or damaged during sectioning.

The aim of this study was to qualitatively study the uptake of single nanoparticles of well-defined size and composition into human skin and to compare these results to conventional microscopy. This also allowed us to develop a protocol that permits the detection of single nanoparticles on ultramicrotome sections of human skin using x-ray microscopy and to investigate nanoparticle penetration on the level of particlemembrane interactions.

\section{Material and Methods}

\subsection{Synthesis and Characterization of Submicron and Nanoparticles}

Gold shell particles were synthesized as suitable sample to provide high contrast in x-ray microscopy. The synthesis approach has been described in detail elsewhere 37 In brief, silica colloids with a diameter of $197 \pm 7 \mathrm{~nm}$ were synthesized using the Stöber method 38 and functionalized with 3 -aminopropyltrimethoxysilane to enable the attachment of small gold nanoclusters (1-2 $\mathrm{nm}$ diam). The gold shell was grown via reduction of an aged solution of chloroauric acid by addition of a hydroxylamine hydrochloride solution.

Gold colloids with a diameter of $42 \pm 3 \mathrm{~nm}$ were synthesized according to the standard sodium citrate reduction method 39 Gold colloids with a diameter of $49 \pm 4 \mathrm{~nm}$ were purchased from Ted Pella Inc. Three silica shells were grown around the particles using a method described elsewhere 40 The first shell served as a spacer between the dye molecules and the metal core. It is necessary to avoid fluorescence quenching of the dye labels 41 The second shell is labeled with fluoreceine isothiocyanate or rhodamine isothiocyanate molecules, as described elsewhere 4243 The outermost layer of pure silica guarantees that the dye labels do not influence the surface properties of the particles. After synthesis, all samples 
were carefully purified by repeated centrifugation and redispersion in water and finally transferred to phosphate buffered saline (PBS) at $\mathrm{pH} 7.4$.

\subsection{TEM}

Size and polydispersity of the particles were determined by TEM. All samples for TEM measurements were prepared by dipping 400 mesh copper grids coated with a thin carbon film or Formvar film (Quantifoil) into dispersions of the particles. TEM images were taken with a Zeiss EM 10 transmission electron microscope with an acceleration voltage of $80 \mathrm{keV}$. The TEM images were analyzed with the software Simple PCI (C-Images).

\subsection{Skin Samples and Nanoparticle Application}

Skin samples from the retroauricular region were obtained from patients undergoing plastic surgery within $1.5 \mathrm{~h}$ after surgery, following written informed consent approved by the Ethics Committee of the Charité-Universitätsmedizin Berlin. Integrity of the skin surfaces was confirmed macroscopically and microscopically (SM33, Hund Wetzlar, Germany). Damaged samples were excluded from the study. The skin was pretreated by one cyanoacrylate skin surface stripping procedure in order to facilitate follicular penetration 5 Subsequently, particles at concentrations of $1-4 \mathrm{mg} / \mathrm{mL}$ in aqueous solution (PBS 7.4) were applied $\left(20 \mu \mathrm{L} / \mathrm{cm}^{2}\right.$ skin) and incubated for $1.5 \mathrm{~h}$ at $37^{\circ} \mathrm{C}$. No additional dispersing agent or surfactant was used. Prior to fixation, adhesive spray (Liquid bandage, Hansaplast) was applied to the skin surface to fixate particles and prevent loss during the prolonged fixation, washing, and embedding processes required for ultramicrotome sectioning.

\subsection{Preparation of Ultramicrotome Sections}

The tissue samples were cut in 1-mm slices by moving an unused razor blade from the dermis side upward to avoid artificial translocation of particles into the tissue. After prefixation in Karnofski media for $30 \mathrm{~min}$, single hair follicles were identified microscopically. Subsequently, $1 \times 2 \times 2$-mm tissue cubes with central hair follicle openings were prepared using microforceps and razor blades. This procedure was followed by fixation in Karnofski media overnight and embedding in epoxy resin. Ultramicrotome sections of 200, 350, and $500 \mathrm{~nm}$ were prepared from each tissue block using an ultramicrotome (Reichert Ultracut S, Leica, Nussloch, Germany, Diamant Knive, Diatome AG, Biel, Switzerland), and collected on 75-mesh, 100-mesh, or 200-mesh (Ni) grids (Agar Scientific Limited, Essex, United Kingdom), respectively.

\subsection{Fluorescence Microscopy of Cryosections}

For conventional fluorescence microscopy, the investigational site of $1 \times 1 \mathrm{~cm}$ was excised from the tissue blocks and split into four squares of $0.5 \times 0.5 \mathrm{~cm}$ each Then, $5-\mu \mathrm{m}$ cryosections were prepared (Cryo-Star HM560 M, Microm International $\mathrm{GmbH}$, Walldorf, Germany) from each block and subjected to fluorescence microscopy (BX60F3, Olympus, Hamburg, Germany), excitation wavelength: 470-490 nm, emission wavelength: $>550 \mathrm{~nm}$.

\subsection{Optical Microscopy for Preselection of Measurement Areas}

Five selected grids or particle holders could be mounted on one microscope holder and could be studied in one series. The thin sections and the particles were screened using a coordinate reading light microscope (Leica DM4000M, equipped with Heidenhain linear encoder) at the Swiss Light Source (SLS) at the Paul-Scherrer-Institute, Villigen, Switzerland. Areas of interest, such as intact stratum corneum and hair follicle openings were searched and the coordinates of the location were recorded. The same coordinates were used for $\mathrm{x}$-ray microscopy experiments.

\subsection{X-Ray Microscope}

The measurements were performed on the PolLux scanning transmission STXM microscope at the SLS. The storage ring runs at $2.4 \mathrm{GeV}$ in the top-up operation mode, which provides a constant electron beam current of $400 \pm 1.5 \mathrm{~mA}$. The PolLux STM uses linearly polarized x-rays from a bending magnet in the photon energy range between 200 and $1200 \mathrm{eV}$ The device is optimized for low background levels and low detection limits. The transmitted photon flux was measured using a photomultiplier tube (Hamamatsu 647P). The STXM microscope provides a flux of $\sim 10^{7}$ photons per second at $300 \mathrm{eV}$. The samples are mounted vertically on a highprecision piezoelectric stage and scanned horizontally and vertically, perpendicular to the x-ray microprobe, which remains fixed, in order to produce a two-dimensional image. Fresnel zone plates are used as focusing optics to generate a submicrometer x-ray probe $(\sim 40 \mathrm{~nm})$ by geometrical demagnification of the exit slit. The transmitted signal is collected by using a small-area detector, consisting of a phosphor screen, which is coupled to a photomultiplier. The image acquisition is performed by computer-controlled processing electronics. The instrument is operated under vacuum $\left(10^{-3} \mathrm{mbar}\right)$ in order to minimize the x-ray absorption by air, which is significant in the soft $\mathrm{x}$-ray regime, as well as sample contamination. Images were recorded at selected photon energies below and in the $\mathrm{C} 1$ s regime $(270-320 \mathrm{eV})$ as well as below and in the $\mathrm{O} 1 \mathrm{~s}$ regime $(500$ and $550 \mathrm{eV})$. The dwell time per point was between 1.2 and $1.6 \mathrm{~ms}$. Image processing was carried out using the aXis2000 software 45

\subsection{Preparation of STXM Samples}

Initially pure samples of the submicron and nanoparticles were investigated by STXM in order to study the X-ray contrast. This is accomplished by applying a droplet of a diluted dispersion of the particles in water on a $100-\mathrm{nm}$ thick $\mathrm{Si}_{3} \mathrm{~N}_{4}$ membrane (Silson Ltd., Northampton, United Kingdom). The optimum conditions for imaging of these particles in skin samples were found by putting droplets of the same particle dispersions at concentrations ranging from $1-4 \mathrm{mg} / \mathrm{mL}$. These were placed subsequently on Ni grids with skin ultramicrotome sections (see above). This yields skin samples that were loaded by high coverages of colloidal submicron and nanoparticles. 


\section{Results}

Initially, the optimum photon energy for imaging human skin samples was determined. STXM measurements were performed in the line-scan mode on ultramicrotome sections of human skin in the C 1 s regime $(280-320 \mathrm{eV})$. Specifically, the transmitted radiation was recorded while a line trajectory was scanned across a part of the sample at each photon energy. The best contrast was observed at $287.3 \mathrm{eV}$, which is located at the $\mathrm{C} 1 \mathrm{~s}$ edge. Further measurements were carried out in the pre-edge regime $(270 \mathrm{eV})$ as well as below and above the $\mathrm{O} 1 \mathrm{~s}$ edge $(500$ and $550 \mathrm{eV})$. The latter proton energies yielded a slightly lower contrast. The X-ray absorption of the particles has been determined from reference data 46 The results indicate that a featureless and only slowly changing absorption cross section occurs for silica $\left(\mathrm{SiO}_{2}\right)$ in the $\mathrm{C} 1 \mathrm{~s}$ regime. The highest absorption occurs below $270 \mathrm{eV}$ (i.e., below the $\mathrm{C} 1 \mathrm{~s}$ edge) 46 Above the $\mathrm{O}$ 1s-edge near $544 \mathrm{eV}$, the absorption is also significantly increased 46 In contrast, particles with an outer gold shell have a very high and nearly structureless absorption in the $\mathrm{C} 1 \mathrm{~s}$ regime $(270-330 \mathrm{eV})$; thus, any energy in this range can be chosen for $\mathrm{x}$-ray microscopy experiments ${ }^{46}$ Their absorption is higher in the $\mathrm{O} 1 \mathrm{~s}$ regime than for silica shell particles. It slightly drops, which is due to the absorbing oxygen in silica above the $\mathrm{O} 1 \mathrm{~s}$ absorption edge 46 Because of their high absorption contrast, the gold-containing particles are excellently suited for tracking in STXM experiments. In spite of their high absorption, absorption saturation was not observed in the present experiments.

First experiments made use of large silica particles $(197 \pm 7 \mathrm{~nm})$ covered by a thick gold shell $(50 \mathrm{~nm})$. TEM measurements showed that the particles (total diameter $=298 \pm 11 \mathrm{~nm}$ ) had a spherical shape and a low polydispersity $(3.8 \%)$ [see Fig. 1(a)]. These particles were precisely imaged on silicon nitride membranes by STXM below the C 1s edge $(270 \mathrm{eV})$, at the $\mathrm{C} 1 \mathrm{~s}$ edge [287.3 eV, see Fig. 1(d)], and above the $\mathrm{O} 1 \mathrm{~s}$ edge $(550 \mathrm{eV})$. Neither in TEM nor in STXM is the core-shell structure of these particles visible because the electron absorption and the photon absorption, respectively, of the shell material is larger than the absorption of the core material 44 The size of the imaged particles in STXM $(\sim 300 \mathrm{~nm})$ is similar to that derived from TEM studies on the same samples.

Small gold cores $(49 \pm 4$ and $42 \pm 3 \mathrm{~nm})$ were used for investigations on the uptake of silica particles in human skin. These particles were coated subsequently by three silica shells, reaching a total diameter of the core-shell particles of $94 \pm 6$ or $161 \pm 13 \mathrm{~nm}$, respectively. The intermediate layers of these shells contained either chemically bound rhodamine (94 $\pm 6 \mathrm{~nm}$ particles) or fluoreceine (161 $\pm 13 \mathrm{~nm}$ particles). The outermost layer was pure silica and prevented an interaction of the dye molecules with the cellular environment (see Material and Methods Section). TEM imaging clearly proved the core-shell structure of the prepared particles [see Figure 1(b) (94 $\pm 6 \mathrm{~nm}$ particles) and Fig. 1(c) (161 $\pm 13 \mathrm{~nm}$ particles)]. The $94 \pm 6 \mathrm{~nm}$ particles could barely be imaged on silicon nitride membranes by STXM. At none of the used photon energies was it possible to obtain a focused image of these particles. Obviously, these particles were at the limit of the spatial resolution of the $\mathrm{x}$-ray microscope during the ex-
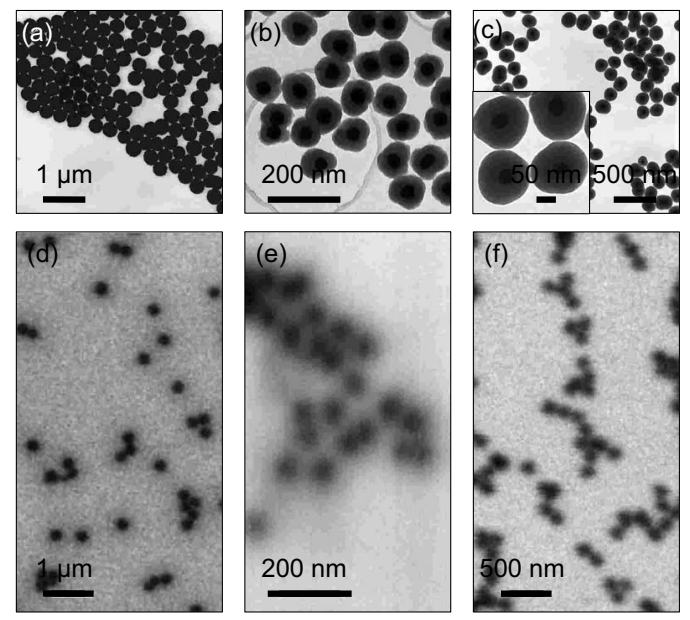

Fig. 1 TEM and STXM images of small colloidal particles on standard grids. TEM and STXM of silica particles $(197 \pm 11 \mathrm{~nm}$ diam) with a gold shell (total diameter $298 \pm 13 \mathrm{~nm}$ ). (a) TEM and (d) STXM recorded at 287.3-eV photon energy, rhodamine-labeled silica particles (total diameter $94 \pm 6 \mathrm{~nm}$ ) with a gold core $(49 \pm 4 \mathrm{~nm}$ diam) (b) TEM and (e) STXM recorded at $270 \mathrm{eV}$ and fluoreceine-labeled silica particles (total diameter $161 \pm 13 \mathrm{~nm}$ ) with a gold core $(42 \pm 3 \mathrm{~nm}$ diam) (c) TEM and (f) STXM recorded at $270 \mathrm{eV}$. Carbon films (TEM) and $\mathrm{Si}_{3} \mathrm{~N}_{4}$ membranes (STXM) are used as substrates, respectively. For better comparison, the magnification of the TEM and STXM images of the same sample is identical. The inset in (C) is a TEM image of the same particles at higher magnification.

periments. Figure 1(d) shows a typical blurred STXM micrograph of these particles at $270 \mathrm{eV}$. In contrast, the $161 \pm 13 \mathrm{~nm}$ gold core particles provided a much better contrast on a silicon nitride membrane in STXM, even though their gold core was smaller $(42 \pm 3 \mathrm{~nm})$ than in the other sample. This sample could be imaged at $270 \mathrm{eV}$ [see Fig. 1(f)] as well as at 287.3 and $550 \mathrm{eV}$. As in the case of the smaller particles, here it was only possible to derive a blurred image.

First experiments with particles in the submicron regime on human skin were carried out with an ultramicrotome section of breast skin (350-nm thickness) treated with a small gold core particle (core: $49 \pm 4 \mathrm{~nm}$, total diameter $94 \pm 6 \mathrm{~nm})$. Subsequently, a droplet of large gold shell particles $(298 \pm 11 \mathrm{~nm}$ diam) was added to this sample. These measurements reveal that at $287.3 \mathrm{eV}$, even the large gold core-shell particles were hardly visible [see Fig. 2(a)]. However, $270 \mathrm{eV}$ appeared to be the favorable photon energy to study gold core or gold shell particles in skin [see Figs. 2(b) and 2(c)] in agreement with the calculated $\mathrm{x}$-ray transmission (see above). Nevertheless, the contrast of the small gold core particles was still low at $270 \mathrm{eV}$. Moreover, after repeated imaging at the same area, small piles of carbon accumulated on the sample, which are a result from radiation damage of the skin. These are caused by prolongated irradiation of a few image points during reinjection in the top-up mode of the storage ring. This could be easily mistaken as evidence for small particles near the resolution limit [see Fig. 2(c)]. Therefore, control measurements were performed above the $\mathrm{O} 1 \mathrm{~s}$ edge $(550 \mathrm{eV})$. In this energy regime it is well known that an enhanced absorption of the outer silica shell occurs 47 Indeed, the STXM micrographs at this energy allowed one to distin- 


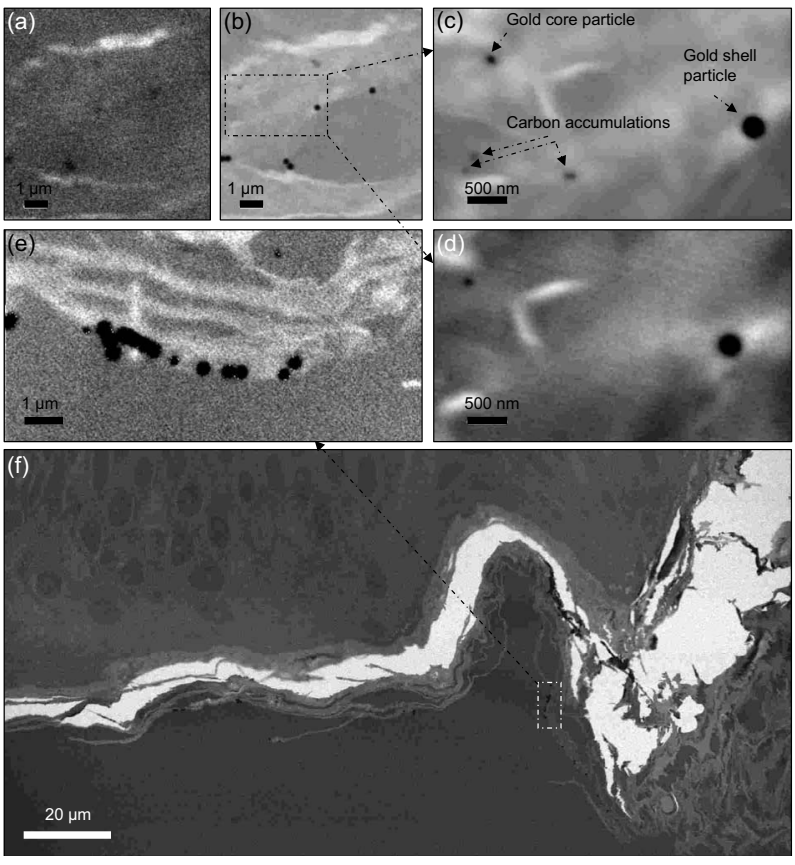

Fig. 2 STXM images of small colloidal particles on human skin at different photon energies. STXM of silica particles $(197 \pm 11 \mathrm{~nm}$ diam $)$ with a gold shell (total diameter $298 \pm 13 \mathrm{~nm}$ ) and rhodamine-labeled silica particles (total diameter $94 \pm 6 \mathrm{~nm})$ with a gold core $(49 \pm 4 \mathrm{~nm}$ diam) on human breast skin recorded at (a) 287.3, (b, c) 270, and (d) $550 \mathrm{eV}$, respectively. The thickness of the ultramicrotome section is $350 \mathrm{~nm}$. (e) corresponds a STXM image of an ultramicrotome section of human breast skin (500-nm thickness) taken after application of $298 \pm 13 \mathrm{~nm}$ gold shell particles recorded at $270-\mathrm{eV}$ photon energy. (f) a larger part of the same section.

guish between small gold core particles and possible artifacts [see Fig. 2(d)]. Furthermore, for the larger gold core particles $(161 \pm 13 \mathrm{~nm}$ diameter), the best contrast was observed at $287.3 \mathrm{eV}$ (see also Figs. 3 and 4. High-resolution STXM of an ultramicrotome section of human breast skin $(500 \mathrm{~nm}$ thickness) taken after application of large gold shell particles

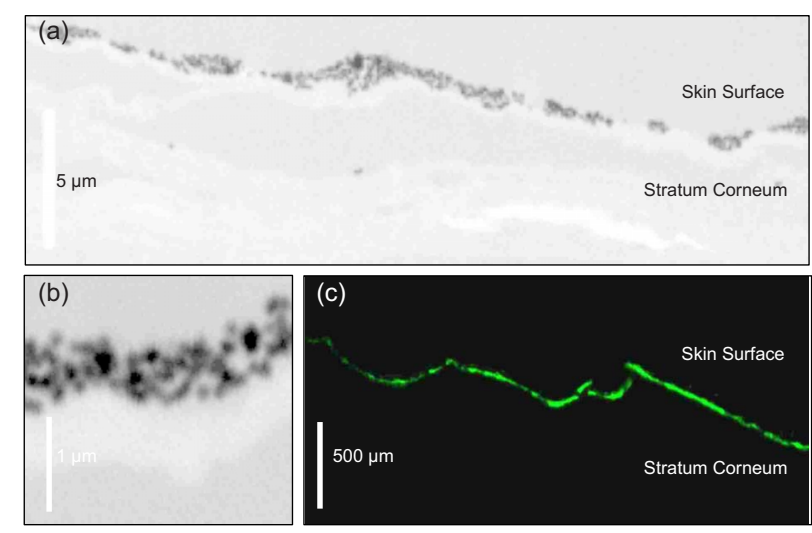

Fig. 3 Penetration of $161 \pm 13 \mathrm{~nm}$ particles in intact human skin. X-ray microscopy images of 350-nm ultramicrotome sections of human skin pretreated with one cyanoacrylate skin surface stripping procedure and $(A, B) 0.1 \% 161 \pm 13 \mathrm{~nm}$ particles $(42 \pm 3 \mathrm{~nm}$ gold core, fluorescein labelling, silica shell) and (C) conventional fluorescence microscopy image of the same sample.
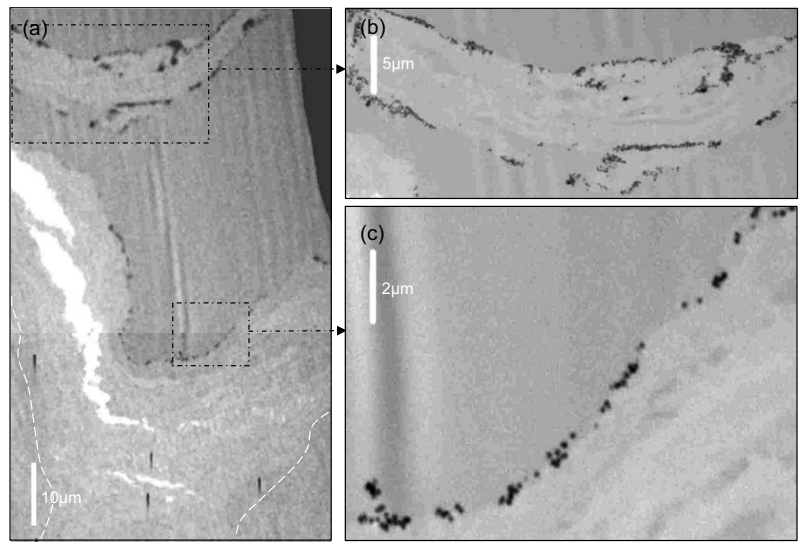

Fig. 4 High-resolution analysis of specific areas of interest. (A) X-ray microscopy micrographs of the penetration of $161 \pm 13 \mathrm{~nm}$ particles in a hair follicle opening. (B, C) For a detailed analysis of penetration processes, selected areas of interest are imaged at high resolution In this superficial compartment of the infundibulum, the particles were spread on the stratum corneum, and no deep penetration was observed.

(298 $\pm 11 \mathrm{~nm}$ diameter) demonstrates that one can distinguish at $270 \mathrm{eV}$ whether aggregated or individual particles are located in the stratum corneum [see Fig. 2(e)]. Note that two gold shell particles appear significantly smaller, about 40$60 \%$ smaller, in this micrograph, although the polydispersity of these particles was rather low $(3.7 \%)$ [see Figs. 1, A) and 1(D)]. Probably these particles were likely sliced through by the ultramicrotome cuts. We present in Fig. 2(f) a larger part of the same ultramicrotome section recorded at $270 \mathrm{eV}$ photon energy. This image clearly shows details of the skin (i.e., the stratum corneum and the underlying keratinocyte layers of the viable epidermis). Among the different tissue sections studied, a thickness of 200-350 nm was identified as an ideal thickness for the detection of the particles used in our investigations. The risk of artificial loss or translocation of particles during preparation and sectioning was crucial for these studies, and sample fragility was also markedly decreased in thicker sections. The contrast of $500 \mathrm{~nm}$ sections, however, although suitable for the investigation of larger particles [e.g., gold shell particles with $298 \pm 11 \mathrm{~nm}$ diameter (see Figure 2(e) ], turned out to be insufficient for the analysis of smaller particles and the identification of cellular structures. In future investigations of smaller particles, however, thinner sections may yield even better images. The sections were mounted on $\mathrm{Ni}$ grids with 75, 100, and 200 mesh. The fixation of the sections on all grids, even with the 75 mesh, was successful. Therefore, the 75- and 100-mesh grids were selected because there is a higher probability that areas of interest are covered by the Ni bars of the thicker 200-mesh grids.

Some $161 \pm 13 \mathrm{~nm}$ particles could conveniently be visualized by STXM according to the developed protocol after optimization of the tissue preparation procedure and addition of spray fixation of the colloidal particles on the skin surface prior to further processing steps. We observed that $161 \pm 13 \mathrm{~nm}$ particles formed somewhat homogeneous layers of single particles on the stratum corneum of the interfollicular epidermis [Fig. 3(a)]. This finding is in accordance with the results obtained from conventional fluorescence micros- 
copy, suggesting the spreading of particles on the skin surface but no deeper penetration into the viable epidermis [Fig. 3(b)]. The high spatial resolution of the STXM measurements, however, allowed us, for the first time, to visualize the location of single particles below $170 \mathrm{~nm}$ diam on and within the stratum corneum [Fig. 3(c)]. STXM imaging of the particle distribution on the surface of a hair-follicle infundibulum revealed lining of particles, occasionally accompanied by trapping of particles between the superficial layers of the stratum corneum (Fig. 4. As observed in interfollicular epidermis, no penetration of the nanoparticles across the stratum corneum was found in these superficial parts of the hair-follicle infundibulum.

\section{Discussion}

The experiments yielded, for the first time, images from x-ray microscopy on the penetration of particles in the size regime between $90 \mathrm{~nm}$ and $300 \mathrm{~nm}$ in human skin samples with the resolution of the single particles. The present experiments indicate that particles with a high x-ray absorption coefficient at photon energies suitable for imaging tissue are needed for the detection at the present resolution limit of STXM. Gold is known for its high absorption contrast in the energy range between 100 and $1000 \mathrm{eV} 31$ The present results show that gold shell silica core particles $(298 \pm 11 \mathrm{~nm}$ diam $)$ yield a high contrast in this energy regime and that silica particles of $161 \pm 13 \mathrm{~nm}$ diam containing an inner gold core of $42 \pm 3 \mathrm{~nm}$ can still be identified as individual particles in human skin samples. Gold core particles with a thinner silica shell (94 $\pm 6 \mathrm{~nm}$ total diameter, $49 \pm 4 \mathrm{~nm}$ gold core) were at the resolution limit of the STXM. They can only be clearly identified, if measurements at different photon energies (below the $\mathrm{C} 1 \mathrm{~s}$ and at the $\mathrm{O} 1 \mathrm{~s}$ edge) are carried out. Further parameters, such as thickness of skin sections, mesh size of the grids, as well as energy levels, were defined (see the Results section) allowing the experimental findings to be reproduced. However, preparation of suitable tissue sections, which fulfill all criteria for reliable measurements, such as avoidance of artificial particle translocation, minimum loss of particles during the preparation procedure, as well as careful sectioning, remain challenging tasks. The present findings clearly indicate that STXM provides detailed images, which have the potential to extend significantly the present knowledge of nanoparticle uptake into skin, which is based on standard experimental techniques. The fact that tissue sections as thick as $350 \mathrm{~nm}$ can be analyzed by STXM is especially valuable for the analysis of nanoparticle-tissue interactions. In contrast, conventional TEM, which can also provide high-resolution structural information for many tissues, requires much thinner ultramicrotome sections of $50-80 \mathrm{~nm}$, as well as additional dehydration and staining 48 This increases the risk of artifacts during the preparation procedure and therefore cannot be applied for the investigation of nanoparticles in the size range of 50-300 nm.

In this study, we show that STXM gives the opportunity to localize the particles in or between the skin cells (e.g., corneocytes). Additional labeling of the gold core particles with a silica shell and fluorescent dyes enabled us to perform with the same samples additional fluorescence microscopy measurements. Hence, the uptake of silica particles in human skin can be studied on identical skin samples using the same type of submicron particles with different complementary imaging techniques (see Fig. 3.

After optimizing the tissue preparation procedure and the addition of spray fixation of the particles on the skin surface prior to further processing steps, layers of $161 \pm 13 \mathrm{~nm}$ particles could conveniently be visualized according to this newly developed experimental protocol. More detailed studies on the penetration of $161 \pm 13 \mathrm{~nm}$ particles on excised human skin revealed that the particles form rather homogeneous layers on the outer skin surface. In contrast to conventional fluorescence microscopy, where the fluorescence appears as a homogeneous band and where the brightness of the fluorescent signal hinders more detailed analysis of the underlying layers of the stratum corneum, STXM enabled us to exclude particle aggregation and demonstrated clearly that penetration of the $161 \pm 13 \mathrm{~nm}$ particles in corneocytes did not occur. Only occasionally did single particles occur underneath the first two layers of the stratum corneum and may also be the result of artificial translocation on the sections. No deeper penetration could be observed in all cases. This finding is complementary to our previous in vitro and in vivo studies on human and porcine skin using different microscopy techniques, including fluorescence microscopy, confocal laser scanning microscopy of tissue sections, Franz cell experiments, and in vivo confocal laser scanning microscopy $\stackrel{4950}{5}$ In addition to these approaches, we recently applied quantitative studies using differential tape stripping, a method developed in our group to monitor and quantify the penetration of fluorescent model compounds in the stratum corneum and hair follicles 51 This approach, where the amount of compound, that penetrates interfollicular epidermis and enters the hair follicle duct are separately quantified, revealed that under physiological conditions, $>95 \%$ of fluorescent model compounds, including fluorescent solid nanoparticles, usually remain in the upper layers of the stratum corneum 52 None of the abovementioned approaches, however, yielded high-resolution images of single nanoparticles. Therefore, the present results from $\mathrm{x}$-ray microscopy represent a highly interesting new approach for penetration studies of skin samples.

The finding that $161 \pm 13 \mathrm{~nm}$ particles do not penetrate the intact stratum corneum is highly relevant because the skin as a barrier organ is especially prone to unintended exposure to environmental hazardous nanoparticles, particulate infectious agents in the nanometer range (e.g., viruses), and to intended exposure (e.g., topical application) of nanoparticle-enriched formulations in cosmetic products, including sunscreens 5354 Our results are in accordance with recent studies by Gamer et al. who found that neither titanium dioxide $<160 \mathrm{~nm}$ nor $80 \mathrm{~nm}$ zink oxide penetrated porcine stratum corneum 55 In accordance with the present results, the above-mentioned previous studies suggest that penetration occurs via shunt penetration (e.g., hair follicles), rather than across the multiple layers of the stratum corneum. Detailed images of single particles below $200 \mathrm{~nm}$ size in combination with the ultrastructural resolution as provided by STXM have not been observed before by using conventional techniques. There is clear evidence that the capacity to penetrate into the viable tissue depends on the size of the particles and that the particle translocation is greatly facilitated in barrier-disrupted or damaged 
skin 51156 The presented results provide a valuable proof concept for the use of STXM in penetration studies. Investigation of smaller nanoparticles and their penetration profiles will follow to visualize penetration processes and particle-cell interactions and to further explore the great potential as well as potentially hazardous effects of particle exposure. In this study, we also found no penetration in superficial compartments of hair follicle openings, where the stratum corneum is still intact. This is consistent with the assumption that particle penetration does most likely occur in deeper parts of the hairfollicle infundibulum, where the differentiation pattern changes from epidermal to trichilemmal differentiation, leading to a physiological disruption of the skin barrier.

Further systematic studies on a series of nanoparticle samples of different size and shape, especially of such particles with a size of $<40 \mathrm{~nm}$, which are expected to penetrate into deeper skin regions, are required to screen the different hair-follicle compartments for nanoparticle penetration processes. We expect from such studies to show that STXM is a powerful tool for probing transport processes in different regions of human skin and tissue.

Furthermore, the use of STXM in other experimental models on skin penetration (e.g., porcine ear skin) may also help elucidate the mechanisms of nanoparticle translocation into the viable tissue. Because thickness of the epidermis, hairfollicle size, and density differ significantly among different species, human explants, as used in this study, are a highly relevant experimental model and have successfully been used by our group to study nanoparticle penetration and cell uptake studies 11 In this model, however, follicular penetration is significantly reduced compared to in vivo conditions, because elastic fibers in the skin contract after excision, leading to an underestimation of the actual penetration, which occurs via hair follicles 57 The additional investigation of penetration in porcine ear, therefore, helps to obtain a more comprehensive view on the penetration, although follicular penetration tends to be overestimated due to the differences in hair size and density. In fact, various studies demonstrated that in vitro investigations on porcine ear skin is well suited as a model for in vivo human skin applying different methods of investigation. These concern, e.g., tape stripping in combination with transepidermal water loss and impedance spectroscopy, 58 light microscopy, transmission electron microscopy, and cryo scanning electron microscopy, 59 studies with diffusion cells, 5960 reconstructed skin 61 penetration studies applying laser scanning microscopy and biopsie 62 as well as the comparison of stratum corneum thickness, diameters of hair and infundibula 63

\section{Conclusions}

In summary, the use of STXM yielded high-resolution images of the distribution of single small particles of $161 \pm 13$ and $298 \pm 11 \mathrm{~nm}$ diam on the skin surface. In the future, detailed ultrastructural and functional studies with emphasis on both cellular uptake and tissue alteration as well as on conformational or chemical changes of nanoparticles in response to skin and tissue exposure will be of importance to further understand the relevance of interactions of nanoscale materials and human tissue on a molecular level. In such studies, STXM may be a key instrument to study the penetration of single nanoparticles with high ultrastructural resolution. Moreover, the chemical selectivity of STXM is advantageous for the identification of any changes on the nanoparticle surface that are caused by uptake and translocation in skin, allowing for a quantitative analysis of the x-ray contrast. Moreover, STXM can also verify spectrally the type of chemical environment where the particles are ending up in the skin. This may allow to identify the mechanisms of their trapping in the skin or their potential impact on health. Further standardization of the procedure and larger series of investigations may allow us to study nanoparticles of different shapes, chemical composition, and surface modification. Furthermore, the importance of different formulations on nanoparticle uptake into skin is expected to be efficiently visualized by STXM, so that the penetration characteristics are modified.

\section{Acknowledgments}

This study was supported by the DFG (SPP 1313 within the Nano-Select network) and the SLS at the Paul-Scherrer Institute (PSI), Villingen (Switzerland). The PolLux end station was financed by the German Federal Ministry of Education and Industry, Contract No. 05 KS4WE1/6. We thank Dr. G. Tzvetkov from the PSI for his support during the experiments at the SLS. We thank Dr. R. Stark and V. Eckert-Funke, Department of Veterinary Anatomy, Freie Universität Berlin (Germany), for the preparation of the ultramicrotome sections and Prof. Dr. R.J. Radlanski and Dr. H. Renz from the Department of Craniofacial Developmental Biology, CharitéUniversitätsmedizin Berlin (Germany) for the use of their electron microscopes.

\section{References}

1. D. B. Warheit, P. J. Borm, C. Hennes, and J. Lademann, "Testing strategies to establish the safety of nanomaterials: conclusions of an ECETOC workshop," Inhalation Toxicol. 19, 631-643 (2007).

2. H. Schaefer and T. Redelmeyer, Skin Barrier: Principles of Percutaneous Absorption. Karger, Basel (1996).

3. J. Lademann, H. Richter, U. F. Schaefer, U. Blume-Peytavi, A. Teichmann, N. Otberg, and W. Sterry, "Hair follicles-a long-term reservoir for drug delivery," Skin Pharmacol. Appl. Skin Physiol. 19, 232-236 (2006).

4. J. Lademann, H. Richter, A. Teichmann, N. Otberg, U. BlumePeytavi, J. Luengo, B. Weiss, U. F. Schaefer, C. M. Lehr, R. Wepf, and W. Sterry, "Nanoparticles-an efficient carrier for drug delivery into the hair follicles," Eur. J. Pharm. Biopharm. 66, 159-164 (2007).

5. R. Toll, U. Jacobi, H. Richter, J. Lademann, H. Schaefer, and U. Blume-Peytavi, "Penetration profile of microspheres in follicular targeting of terminal hair follicles," J. Invest. Dermatol. 123, 168-176 (2004).

6. B. Illel, "Formulation for transfollicular drug administration: some recent advances," Crit. Rev. Ther. Drug Carrier Syst. 14, 207-219 (1997).

7. C. Lauer, L. M. Lieb, C. Ramachandran, G. L. Flynn, and N. D. Weiner, "Transfollicular drug delivery," Pharm. Res. 12, 179-186 (1995).

8. H.-J. Weigmann, J. Ullrich, S. Schanzer, U. Jacobi, H. Schaefer, W. Sterry, and J. Lademann, "Comparison of transepidermal waterloss (TEWL) and spectroscopic absorbance to quantify changes of the stratum corneum after tape stripping," Skin Pharmacol. Appl. Skin Physiol. 18, 180-185 (2005).

9. S. E. Cross, B. Innes, M. S. Roberts, T. Tsuzuki, T. A. Robertson, and P. McCormick, "Human skin penetration of sunscreen nanoparticles: in-vitro assessment of a novel micronized zinc oxide formulation," Skin Pharmacol. Appl. Skin Physiol. 20, 148-154 (2007).

10. B. Baroli, M. G. Ennas, F. Loffredo, M. Isola, R. Pinna, and M. A. Lopez-Quintela, "Penetration of metallic nanoparticles in human fullthickness skin," J. Invest. Dermatol. 127, 1701-1712 (2007). 
11. A. Vogt, B. Combadiere, S. Hadam, K. M. Stieler, J. Lademann, H. Schaefer, B. Autran W. Sterry, and U. Blume-Peytavi, " $40 \mathrm{~nm}$, but not 750 or $1,500 \mathrm{~nm}$, nanoparticles enter epidermal CD1 a+cells after transcutaneous application on human skin," J. Invest. Dermatol. 126, 1316-1322 (2006)

12. L. W. Zhang, N. A. Monteiro-Riviere, "Assessment of quantum dot penetration into intact, tape-stripped, abraded and flexed rat skin," Skin Pharmacol Physiol. 21, 166-180 (2008).

13. J. G. Rouse, J. Yang, J. P. Ryman-Rasmussen, A. R. Barron, and N. A. Monteiro-Riviere, "Effects of mechanical flexion on the penetration of fullerene amino acid-derivatized peptide nanoparticles through skin," Nano Lett. 7, 155-160 (2007).

14. L. E. Meyer, N. Otberg, W. Sterry, and J. Lademann, "In vivo confocal scanning laser microscopy: comparison of the reflectance and fluorescence mode by imaging human skin," J. Biomed. Opt. 11, 044012 (2006)

15. J. Lademann, N. Otberg, H. Richter, L. Meyer, H. Audring, A. Teichmann, S. Thomas, A. Knuttel, and W. Sterry, "Application of optical non-invasive methods in skin physiology: a comparison of laser scanning microscopy and optical coherent tomography with histological analysis," Skin Res. Technol. 13, 119-132 (2007).

16. J. Lademann, H. Weigmann, C. Rickmeyer, H. Barthelmes, H. Schaefer, G. Mueller, and W. Sterry, "Penetration of titanium dioxide microparticles in a sunscreen formulation into the horny layer and the follicular orifice," Skin Pharmacol. Appl. Skin Physiol. 12, 247-256 (1999).

17. A. P. Hitchcock, J. J. Dynes, G. Johansson, J. Wang, and G. Botton, "Comparison of NEXAFS microscopy and TEM-EELS for studies of soft matter," Micron 39, 311-319 (2008).

18. D. Eichert, L. Gregoratti, B. Kaulich, A. Marcello, P. Melpignano, L. Quaroni, and M. Kiskinova, "Imaging with spectroscopic microanalysis using synchrotron radiation," Anal. Bioanal. Chem. 389, 1121-1132 (2007).

19. H. Ade and A. P. Hitchcock, "NEXAFS microscopy and resonant scattering: Composition and orientation probed in real and reciprocal space," Polymer 49, 643-675 (2008).

20. H. Ade, X. Zhang, S. Cameron, C. Costello, J. Kirz, and S. Williams, "Chemical contrast in X-ray microscopy and spatially resolved XANES spectroscopy of organic specimens," Science 258, 972-975 (1992).

21. C. Jacobsen and J. Kirz, "X-ray microscopy with synchrotron radiation," Nat. Struct. Biol. 5, 650-653 (1998).

22. X. Zhang, R. Balhorn, J. Mazrimas, and J. Kirz, "Mapping, and measuring DNA to protein ratios in mammalian sperm head by XANES imaging," J. Struct. Biol. 116, 335-344 (1996).

23. C. Déjugnat, K. Köhler, M. Dubois, G. B. Sukhorukov, H. Möhwald, T. Zemb, and P. Guttmann, "Membrane densification of heated polyelectrolyte multilayer capsules characterized by soft x-ray microscopy," Adv. Mater. (Weinheim, Ger.) 19, 1331-1336 (2007).

24. K. Köhler, C. Déjugnat, M. Dubois, T. Zemb, G. B. Sukhorukov, P. Guttmann, and H. Möhwald, "Soft X-ray microscopy to characterize polyelectrolyte assemblies," J. Phys. Chem. B 111, 8388-8393 (2007).

25. I. Koprinarov, A. P. Hitchcock, W. H. Li, Y. M. Heng, and H. D. H. Stöver, "Quantitative compositional mapping of core-shell polymer microspheres by soft x-ray spectromicroscopy," Macromolecules $\mathbf{3 4}$ 4424-4429 (2001).

26. G. Tzvetkov, B. Graf, R. Wiegner, J. Raabe, C. Quitmann, and R. Fink, "Soft x-ray spectromicroscopy of phase-change microcapsules," Micron 39, 275-279 (2008).

27. J. Stöhr, NEXAFS Spectroscopy, Springer, Berlin (1992).

28. M. G. Anderson, T. Haraszti, G. E. Petersen, S. Wirick, C. Jacobsen, S. W. John, and M. Grunze, "Scanning transmission x-ray microscopic analysis of purified melanosomes of the mouse iris," Micron 37, 689-698 (2006).

29. C. Jacobsen, S. Wirick, G. Flynn, and C. Zimba, "Soft x-ray spectroscopy from image sequences with sub-100 nm spatial resolution," J. Microsc. 197, 173-184 (2000).

30. G. A. Johansson, S. M. Khanna, A. Nair, P. Mannstrom, G. Denbeaux, and M. Ulfendahl, "Exploring the use of soft X-ray microscopy for imaging subcellular structures of the inner ear," J. Microsc. 215, 203-212 (2004).

31. W. Meyer-Ilse, D. Hamamoto, A. Nair, S. A. Lelievre, G. Denbeaux, L. Johnson, A. L. Pearson, D. Yager, M. A. Legros, and C. A. Larabell, "High resolution protein localization using soft x-ray micros- copy," J. Microsc. 201, 395-403 (2001).

32. M. E. Rousseau, D. Hernández Cruz, M. M. West, A. P. Hitchcock, and M. Pezolet, "Nephila clavipes spider dragline silk microstructure studied by scanning transmission x-ray microscopy," J. Am. Chem. Soc. 129, 3897-3905 (2007).

33. A. P. Hitchcock, C. Morin, Y. M. Heng, R. M. Cornelius, and J. L. Brash, "Towards practical soft x-ray spectromicroscopy of biomaterials," J. Biomater. Sci., Polym. Ed. 13, 919-937 (2002).

34. D. Hernández Cruz, M. E. Rousseau, M. M. West, M. Pezolet, and A. P. Hitchcock, "Quantitative mapping of the orientation of fibroinsheets in B. mori cocoon fibers by scanning transmission $\mathrm{X}$-ray microscopy," Biomacromolecules 7, 836-843 (2006).

35. C. J. Buckley N. Khaleque, S. J. Bellamy, M. Robins, and X. Zhang, "Mapping the organic and inorganic components of tissue using NEXAFS," J. Phys. IV 7, 83-90 (1997).

36. A. Mavon, C. Miquel, O. Lejeune, B. Payre, and P. Moretto, "In vitro percutaneous absorption and in vivo stratum corneum distribution of an organic and a mineral sunscreen," Skin Pharmacol. Appl. Skin Physiol. 20, 10-20 (2007).

37. C. Graf and A. van Blaaderen, "Metallodielectric colloidal core-shell particles for photonic applications," Langmuir 18, 524-534 (2002).

38. W. Stöber, A. Fink, and E. Bohn, "Controlled growth of monodisperse silica spheres in the micron size range," J. Colloid Interface Sci. 26, 62-69 (1968).

39. B. V. Enüstün and J. Turkevich, "Coagulation of Colloidal Gold," J. Am. Chem. Soc. 85, 3317-3328 (1963).

40. C. Graf, D. L. J. Vossen, A. Imhof, and A. van Blaaderen, "A general method to coat colloidal particles with silica," Langmuir 19, 66936700 (2003).

41. O. G. Tovmachenko, C. Graf, D. J. van den Heuvel, A. van Blaaderen, and H. C. Gerritsen, "Fluorescence enhancement by metal-core/ silica-shell nanoparticles," Adv. Mater. (Weinheim, Ger.) 18, 91-95 (2006).

42. N. A. M. Verhaegh and A. van Blaaderen, "Dispersions of rhodamine-labeled silica spheres: synthesis, characterization, and fluorescence confocal scanning laser microscopy," Langmuir 10, 1427-1438 (1994)

43. A. Imhof, M. Megens, J. J. Engelberts, D. T. N. de Lang, R. Sprik, and W. L. Vos, "Spectroscopy of fluorescein (FITC) dyed colloidal silica spheres," J. Phys. Chem. B 103, 1408-1415 (1999).

44. C. Degueldre, J. Raabe, G. Kuri, and S. Abolhassani, "Zircaloy-2 secondary phase precipitate analysis by x-ray microspectroscopy," Talanta 75, 402-406 (2008).

45. 〈http://unicorn.mcmaster.ca/aXis2000.html〉 (Access date Mar. 2008, download of software: aXis 2000-Analysis of X-ray Images and Spectra).

46. B. L. Henke, E. M. Gullikson, and J. C. Davis, At. Data Nucl. Data Tables 54, 181-342 (1993).

47. M. Grimm, B. Langer, S. Schlemmer, T. Lischke, U. Becker, W. Widdra, D. Gerlich R. Flesch, and E. Rühl, "Charging mechanisms of trapped element-selectively excited nanoparticles exposed to soft x-rays," Phys. Rev. Lett. 96, 066801 (2006).

48. B. A. Afzelius and A. B. Maunsbach, "Biological ultrastructure research; the first 50 years," Tissue Cell 36, 83-94 (2004).

49. U. Jacobi, H. Taube, U. F. Schäfer, W. Sterry, and J. Lademann, "Comparison of four different in vitro systems to study the reservoir capacity of the stratum corneum," J. Controlled Release 103, 61-71 (2005).

50. J. Lademann, N. Otberg, H. Richter, L. Meyer, H. Audring, A. Teichmann, S. Thomas, A. Knuettel, and W. Sterry, "Application of optical non-invasive methods in skin physiology: a comparison of laser scanning microscopy and optical coherent tomography with histological analysis," Skin Res. Technol. 13, 119-132 (2007).

51. M. Ossadnik, V. Czaika, A. Teichmann, W. Sterry, H. J. Tietz, J. Lademann, and S. Koch, "Differential stripping: introduction of a method to show the penetration of topically applied antifungal substances into the hair follicles," Mycoses 50, 457-462 (2007).

52. A. Teichmann, U. Jacobi, M. Ossadnik, H. Richter, S. Koch, W Sterry, and J. Lademann, "Differential stripping: determination of the amount of topically applied substances penetrated into the hair follicles," J. Invest. Dermatol. 125, 264-269 (2005).

53. S. Singh and H. S. Nalwa, "Nanotechnology and health safetytoxicity and risk assessments of nanostructured materials on human health," J. Nanosci. Nanotechnol. 7, 3048-3070 (2007).

54. S. T. Stern and S. E. McNeil, "Nanotechnology safety concerns re- 
visited," Toxicol. Sci. 101, 4-21 (2008).

55. A. O. Gamer, E. Leibold, and B. van Ravenzwaay, "The in vitro absorption of microfine zinc oxide and titanium dioxide through porcine skin," Toxicol. In Vitro 20, 301-307 (2006).

56. A. Rolland, N. Wagner, A. Chatelus, B. Shroot, and H. Schaefer, "Site-specific drug delivery to pilosebaceous structures using polymeric microspheres," Pharm. Res. 10, 1738-1744 (1993).

57. A. Patzelt, H. Richter, R. Buettemeyer, H. J. Roewert Huber, U. Blume-Peytavi, W. Sterry, and J. Lademann, "Differential stripping demonstrate a significant reduction of the hair follicle reservoir in vitro compared to in vivo," Eur. J. Pharm. Biopharm. 70, 234-238 (2008).

58. N. Sekat, Y. N. Kalia, R. H. Guy, "Biophysical study of porcine ear skin in vitro and its comparison to human skin in vivo," J. Pharm. Sci. 19, 2376-2381 (2002).

59. W. Meyer, J. Kacza, N. H. Zschemisch, S. Godynnicki, and J. Seeger, "Observations on the actual structural conditions in the stratum cor- neum superficial dermidis of porcine ear skin, with special reference to its use as model for human skin," Ann. Anat. Pathol. (Paris) 189, 143-156 (2007).

60. S. Andega, N. Kanikkannan, and M. Singh, "Comparison of the effect of fatty alcohols on the permeation of melatonin between porcine and human skin," J. Controlled Release 77, 17-25 (2001).

61. A. Mahmoud, A. Haberland, M. Dürrfeld, D. Heydeck, S. Wagner, and M. Schaefer-Korting, "Cutaneous estradiol permeation, penetration, and metabolism in pig and man," Skin Pharmacol. Appl. Skin Physiol. 18, 27-35 (2005).

62. U. Jacobi, R. Toll, W. Sterry, and J. Lademann, "Do follicles play a role as penetration pathways in in vitro studies on porcine skin?-an optical study," Laser Phys. 15, 1594-1598 (2005).

63. U. Jacobi, M. Kaiser, R. Toll, S. Mangelsdorf, H. Audring, N. Otberg, W. Sterry, and J. Lademann, "Porcine ear skin: an in vitro model for human skin," Skin Res. Technol. 13, 19-24 (2007). 